\title{
Protein Secondary Structure Prediction using an Optimised Bayesian Classification Neural Network
}

\author{
Son T.Nguyen, Colin G.Johnson \\ School of Computing, University of Kent, Canterbury, Kent, United Kingdom \\ \{S.Thanh-Nguyen, C.G.Johnson\}@kent.ac.uk
}

Keywords: Protein secondary structure prediction, classification Bayesian neural network, optimised network architecture.

\begin{abstract}
The prediction of protein secondary structure is a topic that has been tackled by many researchers in the field of bioinformatics. In previous work, this problem has been solved by various methods including the use of traditional classification neural networks with the standard error back-propagation training algorithm. Since the traditional neural network may have a poor generalisation, the Bayesian technique has been used to improve the generalisation and the robustness of these networks. This paper describes the use of optimised classification Bayesian neural networks for the prediction of protein secondary structure. The well-known RS126 dataset was used for network training and testing. The experimental results show that the optimised classification Bayesian neural network can reach an accuracy greater than $75 \%$.
\end{abstract}

\section{INTRODUCTION}

The accurate prediction of protein secondary structure is an important step to understand protein folding. A large number of papers have tackled this problem, with the most common approach being the use of various machine learning methods to learn the connection between amino acid sequences and secondary structure. Some of these approaches use the amino acid states directly as the input data to learning, whereas other methods have used the biophysical features of amino acids, sequence homology, pattern matching and statistical analyses of proteins of known structures (Rost and Sander, 1993a), (Rost and Sander, 1993b).

The standard way of presenting this task as a machine learning problem is as a classification problem, where each data instance consists of a number of predictor features (e.g. the neighbouring amino acid values) and a class drawn from the set $\{$ helix, strand, coil\}. The aim of the learned model is to be able to predict this class for examples not seen during training.

According to (Holley and Karpus, 1989), the maximum accuracy of predicting three states (helices, strands and coils) has a limit, due to the amount of data available and/or that the secondary structure is determined by tertiary interactions not included in the local sequence. Nonetheless, models have been learned that can predict the class with reasonable accuracy ( $65 \%$ for simple methods, rising to around $92 \%$ for more sophisticated methods that use additional data about similar proteins) (Lee et al., 2012)

This paper describes the use of classification Bayesian neural networks for the prediction of protein secondary structure. In the past, classification Bayesian neural networks have been proven to be useful for several classification tasks (Nguyen at all, 2004), (Nguyen at all, 2006), (Penny and Robert, 1999) and (Thodberg, 1996). Unlike the traditional neural network training, the Bayesian neural network training does not require a validation set separated from the training subset. As a result, all of the available data set can be divided into only two subsets: the training subset and the test subset (Mackay, 1992a), (Mackay, 1992b). The Bayesian neural network training also encourages generalisation as the values of the weight decay parameters, sometimes known hyper-parameters, can be well adjusted during the network training 
phase. Moreover, Bayesian neural networks allow users to rank and compare different networks with different architectures. Therefore, the optimal network architecture can be easily found based on evaluating the log evidence of candidate networks with the Bayesian framework (Penny, 1999), (Mackay, 1992b).

The structure of the paper is organised as follows. Section II provides an overview of protein secondary structure prediction and classification neural networks. In Section III, the formulation of classification Bayesian neural networks is briefly described. In Section IV, the paper gives the assessment methods for the obtained results. Section $\mathrm{V}$ presents how to train and optimise classification Bayesian neural networks for predicting three states: helices, strands and coils. Finally, Section VI provides a conclusion.

\section{PROTEIN SECONDARY STRUCTURE PREDICTION AND CLASSIFICATION NEURAL NETWORKS}

Protein structure prediction is the foundation of protein structure biology. Proteins are macromolecules made of chains of 20 different amino acids, which fold into a particular threedimensional structure that is distinctive to that protein. This three-dimensional structure is what determines the function of a protein. The ultimate goal is to understand the function of proteins, and therefore an important step towards this understanding is to understand the protein structure and how this relates to its sequence. Biochemists distinguish four distinct aspects of a protein's structure: Primary structure, Secondary structure, Tertiary structure and Quaternary structure. Protein Secondary Structure Prediction (PSSP) means predicting which parts of a protein will form the large-scale structures known as $\alpha$-helix, $\beta$-strand and coils, based on the amino acid sequence of a protein (Mottalib et al, 2010).

In the last two decades, a huge number of approaches have been taken to the PSSP. In these works, the probabilistic approaches were the first to be used. The first attempt at using neural networks for PSSP was done by Qian and Sejnowski in 1988, and they obtained an accuracy of $64.3 \%$ (Qian and Sejnowski, 1988). More recent neural network based approaches have achieved accuracies greater than 70\% (Rost and Sander, 1993a), (Jones, 1999). The most important improvement in these approaches is to modify the input set to the neural network by finding similar proteins from a large database, and forming an input based on the proportion of amino acid values at each position in the sequence. The aim of this is to provide more information to the network about the kind of protein, and to eliminate the influence of an uncharacteristic amino acid at a particular position.

This part of the paper will describe how classification neural networks are used for PSSP. The primary sequences are used as the network input. In order to read the input, a moving window through the sequences needs to be created. According to (Qian and Sejnowski, 1988), the size of the moving window should be chosen to be 13 as this window size has given the best performances when testing the trained network on the test subsetthis window size has also been found in many subsequent papers.

In this work, the define secondary structure of proteins (DSSP) method is used. According to this method, the secondary structure of each residue classifies into 8 classes, namely $\mathrm{H}$ ( $\alpha$-helix), G (310helix), I ( $\pi$-helix), B (isolated $\beta$-bridge), E (extended $\beta$-strand), T (hydrogen bonded turn), $S$ (bend), and $\mathrm{C}$ (not HBEGIT or S). The prediction methods are assessed for only 3 standard classes associated with $\alpha$ helices (H), $\beta$-strands (E) and coils (C). Hence, 8 classes are reduced to 3 . In the literature, there are four main mappings to perform the reduction process (Sepideh et al., 2008). These are:

1. $\mathrm{H}, \mathrm{G} \rightarrow \mathrm{H}$

$$
\mathrm{E} \rightarrow \mathrm{E}
$$$$
\mathrm{S}, \mathrm{T}, \mathrm{B}, \mathrm{I}, \mathrm{C} \rightarrow \mathrm{C}
$$

2. $\mathrm{H} \rightarrow \mathrm{H}$

$\mathrm{E} \rightarrow \mathrm{E}$

$\mathrm{G}, \mathrm{S}, \mathrm{T}, \mathrm{B}, \mathrm{I}, \mathrm{C} \rightarrow \mathrm{C}$

3. $\mathrm{H}, \mathrm{G}, \mathrm{I} \rightarrow \mathrm{H}$

$\mathrm{E}, \mathrm{B} \rightarrow \mathrm{E}$

$\mathrm{S}, \mathrm{T}, \mathrm{C} \rightarrow \mathrm{C}$

4. $\mathrm{H}, \mathrm{G} \rightarrow \mathrm{H}$

$\mathrm{E}, \mathrm{B} \rightarrow \mathrm{E}$

$\mathrm{S}, \mathrm{T}, \mathrm{I}, \mathrm{C} \rightarrow \mathrm{C}$

Here, the method 2 is adopted in this research as it is considered as the strictest criterion. In order to encode the secondary structure classes for the classification, three output units are assigned in our neural network as binary values as follows

$\mathrm{H}=[1,0,0], \mathrm{E}=[0,1,0]$, and $\mathrm{C}=[0,0,1]$ 


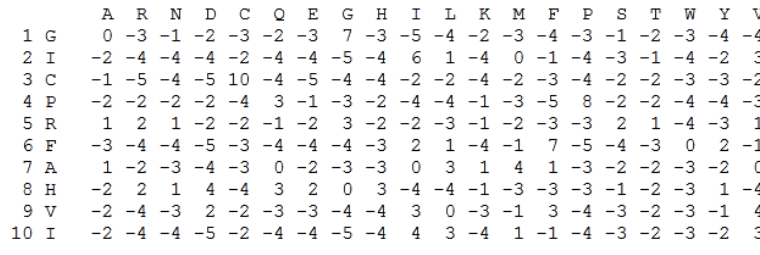

(a)

$\begin{array}{lllllllll}0.50000000 & 0.04742587 & 0.26894142 & 0.11920292 & 0.04742587 & 0.11920292\end{array}$ $\begin{array}{lllllllll}0.11920292 & 0.01798621 & 0.01798621 & 0.01798621 & 0.11920292 & 0.0179862\end{array}$ $\begin{array}{lllllllll}0.26894142 & 0.00669285 & 0.01798621 & 0.00669285 & 0.99995460 & 0.0179862\end{array}$ $\begin{array}{lllllllll}0.11920292 & 0.11920292 & 0.11920292 & 0.11920292 & 0.01798621 & 0.9525741\end{array}$ $\begin{array}{lllllllllllllllll}0.73105858 & 0.88079708 & 0.73105858 & 0.11920292 & 0.11920292 & 0.26894142\end{array}$ $\begin{array}{llllllll}0.04742587 & 0.01798621 & 0.01798621 & 0.00669285 & 0.04742587 & 0.0179862\end{array}$ $\begin{array}{llllllll}0.73105858 & 0.11920292 & 0.04742587 & 0.01798621 & 0.04742587 & 0.5000000\end{array}$ $\begin{array}{lllllll}0.11920292 & 0.88079708 & 0.73105858 & 0.98201379 & 0.01798621 & 0.95257413\end{array}$ $\begin{array}{llllll}0.11920292 & 0.01798621 & 0.04742587 & 0.88079708 & 0.11920292 & 0.0474258\end{array}$ $\begin{array}{llllll}0.11920292 & 0.01798621 & 0.01798621 & 0.00669285 & 0.11920292 & 0.0179862\end{array}$ $\begin{array}{llllllll}0.88079708 & 0.11920292 & 0.95257413 & 0.11920292 & 0.04742587 & 0.11920292\end{array}$ (b)

Figure 1: The PSSM matrix: (a) the raw matrix values, (b) the matrix values normalised with the standard logistic function.

The prediction of protein secondary structure based on a multiple alignment profile of protein instead of a single sequence has been adopted as a way to improve the prediction accuracy in several papers (Rost and Sander, 1993a), (Rost and Sander, 1993b). The aim of this is to improve predictive power by finding proteins that are similar to the protein being examined, and modifying the input to be a mixture of the original protein information and that of these similar proteins.

There are two kinds of multiple alignment profiles: 1) the multiple sequence alignment profiles (MSAP), 2) the position-specific score matrices (PSSM) (Sepideh at al, 2008). In this research, the PSSMs for the RS126 dataset are used and they can be easily obtained from (Jpred 3), which is a simple and accurate protein secondary structure prediction method incorporating two feed-forward neural networks. The profile matrix has $20 x L$ elements, where $\mathrm{L}$ is the length of the target sequence as shown in Figure.1. As the values of the feedforward neural network inputs can only accept 0 to 1, the raw PSSM needs to be normalised using the logistic function as shown in equation (1).

$$
f(x)=\frac{1}{1+e^{-x}}
$$

where $x$ is the raw matrix value.

As the window size is 13 with 20 amino acids, there are $13 \times 20+1=261$ neural network inputs (the last input for the bias term). There are three output units corresponding to three states (helix, strand and other structures (sometimes known as loops or coils)). The network has a single hidden layer, as one hidden layer is sufficient to solve every problem including regression and classification (Bishop, 1995). The optimal number of hidden nodes is theoretically determined based on the Bayesian inference discussed in details in Section III.

\section{CLASSIFICATION BAYESIAN NEURAL NETWORKS}

Bayesian learning of multi-layer perceptron neural networks is performed by considering Gaussian probability distributions of the weights which give the best generalization (Mackay, 1992a), (Mackay, 1992b). In particular, the weights $w$ in network $X$ are adjusted to their most probable values given the training data $D$. Specifically, the posterior distribution of the weights can be computed using Bayes' rule as follows

$$
p(w \mid D, X)=\frac{p(D \mid w, X) p(w \mid X)}{p(D \mid X)}
$$

where $p(D \mid w, X)$ is the likelihood function, which contains information about the weights from observations, and the prior distribution $p(w \mid X)$ contains information about the weights from background knowledge. The denominator, $p(D \mid X)$, is known as the evidence for network $X$ given dataset $D$.

Given a set of candidate networks $X_{i}$ having different numbers of hidden nodes, the posterior probability of each network can be expressed as

$$
p\left(X_{i} \mid D\right)=\frac{p\left(D \mid X_{i}\right) p\left(X_{i}\right)}{p(D)}
$$

If the networks are assumed to be equally probable before any data is observed, then $p\left(X_{i}\right)$ is the same for all networks. Since $p(D)$ does not depend on the network, then the probable network is the one with the highest evidence $p\left(D \mid X_{i}\right)$. Therefore, the evidence can be used to compare and rank different candidate networks.

The weights and biases of the Bayesian neural network are grouped into a single vector and determined as follows 


$$
w_{m+1}=w_{m}+\alpha_{m} d_{m}
$$

where $w_{m+1}$ is the weight vector at the training iteration $m+1, w_{m}$ is the weight vector at the training iteration $m, \alpha_{m}$ is the adaptive learning rate at the training iteration $m$ and $d_{m}$ is the search direction at the training iteration $m$. The adaptive learning rate is adjusted during the training phase based on the Scaled Conjugate Gradient method (Moller, 1993). In this research, the search direction is predefined to be the negative gradient $-g_{m}$.

Regularisation is used to prevent any weights becoming excessively large, which can lead to poor generalisation. For a multi-layer perceptron neural network classifier with $G$ groups of weights and biases, a weight decay penalty term proportional to the sum of squares of the weights and biases is added to the data error function $E_{D}$ to obtain the cost function

$$
\begin{gathered}
S=E_{D}+\sum_{g=1}^{G} \xi_{g} E_{W_{g}} \\
E_{W_{g}}=\frac{1}{2}\left\|w_{g}\right\|^{2} \quad(g=1, \ldots, G)
\end{gathered}
$$

where $S$ is called the cost function, $\xi_{g}$ is a nonnegative scalar, sometimes known as a hyperparameter, ensuring the distribution of weights and biases in group $g$ and $w_{g}$ is the vector of weights and biases in group $g$.

In network training, the hyperparameters are initialised to be arbitrary small values. The cost function is then minimised using the Scaled Conjugate Gradient method. When the cost function has reached a local minimum, the hyperparameter $\xi_{g}(g=1, \ldots, G)$ is re-estimated. This task requires computing the Hessian matrix of the cost function:

$$
A=H+\sum_{g=1}^{G} \xi_{g} I_{g}
$$

where $H$ is the Hessian matrix of $E_{D}$ and $I_{g}$ is the identity matrix, which selects weights in the $g$ th group. The number of 'well-determined' weights $\gamma_{g}$ in group $g$ is calculated based on the old value of $\xi_{g}$ as follows:

$$
\gamma_{g}=W_{g}-\xi_{g} \operatorname{tr}\left(A^{-1} I_{g}\right) \quad(g=1, \ldots, G)
$$

The new value of the hyperparameter $\xi_{g}$ is then re-estimated as

$$
\xi_{g}=\frac{\gamma_{g}}{2 E_{W_{g}}} \quad(g=1, \ldots, G)
$$

The hyperparameters need to be re-estimated several times until the cost function value ceases to change significantly between consecutive reestimation periods. After the network training is completed, the values of parameters $\gamma_{g}$ and $\xi_{g}$ are then used to compute the $\log$ evidence of network $X_{i}$ having $M$ hidden nodes as follows (Penny and Robert, 1999):

$$
\begin{aligned}
\ln E \mathcal{V}\left(X_{i}\right) & =-S+\sum_{g=1}^{G} \frac{W_{g}}{2} \ln \xi_{g}-\frac{1}{2} \ln |A|+\ln M !+M \ln 2 \\
& +\sum_{g=1}^{G} \frac{1}{2}\left(\frac{4 \pi}{\gamma_{g}}\right)-G \ln (\ln \Omega)
\end{aligned}
$$

where $W_{g}$ is the number of weights and biases in group $g$, and $\Omega$ is set to be $10^{3}$ (Thodberg, 1996). However, $\Omega$ is a minor factor because it is the same for all models and therefore does not effect to the relative comparison of log evidence of different network architectures. Equation (10) is used to compare different networks having different numbers of hidden nodes. The best network will be selected with the highest log evidence.

\section{EVALUATION METHODS}

A useful accuracy evaluation for classification neural networks is well-known three state overall residue accuracy percentage defined as follows

$$
Q_{3}=\frac{P_{\alpha}+P_{\beta}+P_{\text {loop }}}{N} \times 100
$$


where $P_{\alpha}, P_{\beta}$ and $P_{\text {loop }}$ are the number of correctly predicted $\alpha$-helix, $\beta$-sheet and loop, respectively. $N$ is the total number of residues in a given protein sequence.

Another widely used accuracy measurement is the Matthew's correlation coefficients. In the case of $P_{\alpha}$ helix, this coefficient is determined as follows

$$
C_{\alpha}=\frac{p_{\alpha} n_{\alpha}-u_{\alpha} o_{\alpha}}{\sqrt{\left(n_{\alpha}+u_{\alpha}\right)\left(n_{\alpha}+o_{\alpha}\right)\left(p_{\alpha}+u_{\alpha}\right)\left(p_{\alpha}+o_{\alpha}\right)}}
$$

where $p_{\alpha}$ is the number of correctly predicted positive cases, $n_{\alpha}$ is the number of correctly rejected negative cases, $o_{\alpha}$ is the number of overpredicted cases (false positives), and $u_{\alpha}$ is the number of under-predicted cases (misses). Similarly, $C_{\beta}$ and $C_{\text {loop }}$ can be also defined for $\beta$-sheet and loop, respectively. If the coefficients are equal to 1 , the model predictions are $100 \%$ correct. Whereas, if the coefficients are equal to -1 , the model predictions are $100 \%$ incorrect.

\section{EXPERIMENTS AND RESULTS}

The RS126 dataset was used for training and testing the networks (Rost and Sander, 1993a). All of the dataset was randomly divided into seven subsets. A seven fold cross-validation technique was applied to determine the prediction accuracy. In particular, six subsets were used for training networks and the remaining subset was used for testing networks. This procedure was repeated for the different test subsets. Bayesian neural networks with different numbers of hidden nodes were trained to select the optimal network architecture. These networks have the following specification, as discussed in detail earlier in the paper:

- four hyperparameters $\xi_{1}, \xi_{2}, \xi_{3}$ and $\xi_{4}$ to constrain the magnitudes of the weights on the connection from the input nodes to the hidden nodes, the biases of the hidden nodes, the weights on the connection from the hidden nodes to the output nodes, and the biases of the output nodes;
- 261 inputs, corresponding to 20 inputs for each letter in the moving with the size is 13 and one bias term with a constant value of 1 ;

- three outputs, each corresponding to one of the states: helix, strand and other structures.

For a given number of hidden nodes, five networks with different initial values of the weights and biases were trained. The training procedure was implemented as follows:

1. The weights and biases in four different groups were initialised by random selections from zeromean, unit variance Gaussians and the initial hyperparameters were chosen to be small values.

2. The network was trained to minimise the cost function $S$ using Scaled Conjugate Gradient training algorithm.

3. When the network training had reached a local minimum, the values of the hyperparameters were re-estimated according to equation (8) and (9).

4. Steps 2 and 3 were repeated until the cost function value was smaller than a predetermined value and did not change significantly in subsequent re-estimations.

The performances of the trained networks were tested on the seventh subset. As shown in Figure 2, the networks having four hidden nodes is the last increase that produces a meaningful increase in $\log$ evidence. This means that four hidden nodes are sufficient to solve the problem. Table 1 shows the change of hyperparameters according to the periods of re-estimation of a specific network training run. For each period, there are 100 predefined trainingiterations.

Table 2 shows the prediction accuracy and the Matthew's correlation coefficients on three states from the classification Bayesian neural network. We can see that the accuracy is $75.77 \%$. Next, a standard classification neural network that has the same structure with the classification Bayesian neural network was trained to obtain the three-state prediction accuracy. However, the prediction accuracy of the trained classification standard neural network $74.97 \%$ and the Matthew's correlation coefficients, shown in Table 3, are also smaller than those of the trained classification Bayesian neural network shown in Table 2. Whilst this increase is small, progress in this area has typically come from 


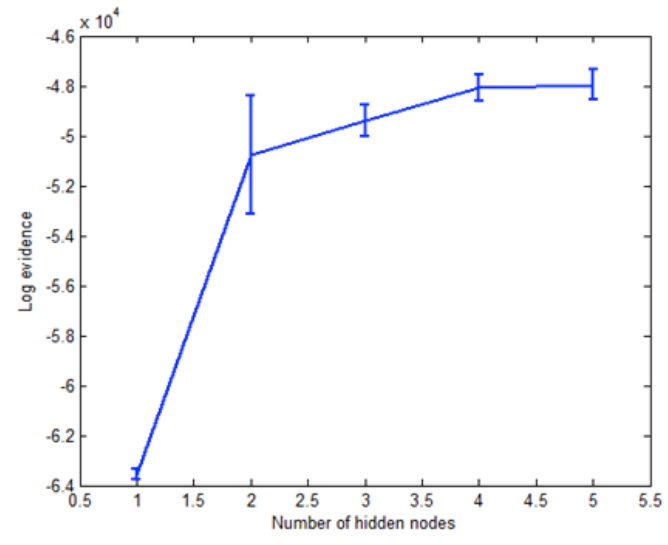

Figure 2: Log evidence versus number of hidden nodes: The solid curve shows the evidence averaged over the five networks.

the accumulation of small improvements, which can be combined together to make a larger improvement.

\section{CONCLUSIONS}

The results obtained show that Bayesian neural networks can be used to predict the protein secondary structure with the maximum accuracy of $75.77 \%$. This is better than the traditional neural network training methods. According to the obtained results, the use of four hidden nodes is an optimal choice for the network architecture. This number of hidden nodes can give the best generalisation of the trained network without the use of a validation set. Therefore, the available data was only divided into two subsets: one for training and another for testing. Moreover, Bayesian training for neural network can automatically adjust the hyperparameters during the training phase.

The procedure for determining the optimal structure of the classification standard neural network (the growing and pruning technique) has not been mentioned in this paper as this approach requires a lot of statistical tasks. The main disadvantage of the Bayesian learning for feedforward neural networks is that it takes a quite long time on evaluating the Hessian matrix, especially when the number of network parameters (weights and biases) is relatively large.
Table 1: The change of hyperparameters according to the periods of re-estimation.

\begin{tabular}{|c|c|c|c|c|}
\hline Periods & $\xi_{1}$ & $\xi_{2}$ & $\xi_{3}$ & $\xi_{4}$ \\
\hline 1 & 31.392 & 1.371 & 0.529 & 0.775 \\
\hline 2 & 99.919 & 2.389 & 0.334 & 2.432 \\
\hline 3 & 198.498 & 4.055 & 0.231 & 3.949 \\
\hline
\end{tabular}

Table 2: The three-state prediction accuracy and Matthew's correlation coefficients of classification Bayesian neural network.

\begin{tabular}{|c|c|c|c|c|}
\hline & & \multicolumn{3}{|c|}{$\begin{array}{c}\text { Matthew's Correlation } \\
\text { Coefficients }\end{array}$} \\
\hline Fold & $Q_{3}(\%)$ & $C_{\alpha}$ & $C_{\beta}$ & $C_{\text {loop }}$ \\
\hline A & 75.840 & 0.699 & 0.531 & 0.565 \\
\hline B & 78.187 & 0.728 & 0.607 & 0.604 \\
\hline C & 72.422 & 0.635 & 0.510 & 0.524 \\
\hline D & 75.319 & 0.658 & 0.550 & 0.540 \\
\hline E & 74.826 & 0.641 & 0.580 & 0.542 \\
\hline F & 76.362 & 0.697 & 0.598 & 0.569 \\
\hline G & 77.462 & 0.696 & 0.578 & 0.604 \\
\hline Average & $\mathbf{7 5 . 7 7 4}$ & $\mathbf{0 . 6 7 9}$ & $\mathbf{0 . 5 6 5}$ & $\mathbf{0 . 5 6 4}$ \\
\hline
\end{tabular}

Table 3: The three-state prediction accuracy and Matthew's correlation coefficients of standard classification Bayesian neural network.

\begin{tabular}{|c|c|c|c|c|}
\hline & & \multicolumn{3}{|c|}{$\begin{array}{c}\text { Matthew's Correlation } \\
\text { Coefficients }\end{array}$} \\
\hline Fold & $Q_{3}(\%)$ & $C_{\alpha}$ & $C_{\beta}$ & $C_{\text {loop }}$ \\
\hline $\mathrm{A}$ & 75.927 & 0.689 & 0.543 & 0.565 \\
\hline $\mathrm{B}$ & 77.347 & 0.725 & 0.583 & 0.587 \\
\hline $\mathrm{C}$ & 71.321 & 0.619 & 0.496 & 0.502 \\
\hline $\mathrm{D}$ & 74.826 & 0.646 & 0.551 & 0.536 \\
\hline $\mathrm{E}$ & 73.812 & 0.622 & 0.571 & 0.521 \\
\hline $\mathrm{F}$ & 74.739 & 0.669 & 0.572 & 0.548 \\
\hline $\mathrm{G}$ & 76.796 & 0.669 & 0.568 & 0.604 \\
\hline Average & $\mathbf{7 4 . 9 6 7}$ & $\mathbf{0 . 6 6 3}$ & $\mathbf{0 . 5 5 5}$ & $\mathbf{0 . 5 5 2}$ \\
\hline
\end{tabular}




\section{REFERENCES}

B.Rost and C.Sander, "Prediction of protein secondary structure at better than 70\% accuracy," J.Mol.Biol.,vol. 232, pp. 584-599, 1993a.

B. Rost and C. Sander, "Improved prediction of protein secondary structure by use of sequence profiles and neural networks.," Proc, Natl, Acad, Sci, Biophysics, USA, pp. 7558 - 7562, 1993 b.

L. H. Holley and M. Karpus, "Protein secondary structure prediction with a neural network," Proc, Natl, Acad, Sci, Biophysics, USA, vol. 86, pp. 152 - 156, 1989.

L. Lee, J. L. Leopold, and R. L. Frank, "Protein secondary structure prediction using BLAST and exhaustive RTRICO, the search for optimal segment length and threshold," 2012 IEEE Symposium on Computational Intelligence in Bioinformatics and Computational Biology (CIBCB), pp. $35-42,2012$.

S.T.Nguyen, H.T.Nguyen, and P. Taylor, "Hands-Free Control of Power Wheelchairs using Bayesian Neural Networks," Proceedings of IEEE Conference on Cybernetics and Intelligent Systems, Singapore, 2004, pp. $745-749,2004$.

S. T. Nguyen, H.T.Nguyen, P. Taylor, and J. Middleton, "Improved Head Direction Command Classification using an Optimised Bayesian Neural Network," Proceedings of IEEE International Conference of the Engineering in Medicine and Biology Society, New York City, New York, USA, August 30-Sept. 3, 2006.

W.D. Penny and S. J. Roberts, "Bayesian neural networks for classification: how useful is the evidence framework," Neural Networks, vol. 12, pp. 877 - 892, 1999.

H. H. Thodberg, "A review of Bayesian neural networks with an application to near infrared spectroscopy," IEEE Transactions on Neural Networks, vol. 7, pp. 56 - 72, 1996.

D. MacKay, "A practical Bayesian Framework for Backpropagation Networks," Computation and Neural Systems, vol. 4, pp. 448-472, 1992a.

D. MacKay, "The Evidence Framework Applied to Classification Networks," Neural Computation, vol. 4, pp. $720-736,1992 b$.

C. M. Bishop, "Neural networks for pattern recognition," Oxford: Clarendon Press; New York: Oxford University Press, 1995.

M. A. Mottalib, M. S. R. Mahdi, A. B. M. Z. Haque, S. M. A. Mamun, and H. A. Al-Mamun, "Protein Secondary Structure Prediction using Feed-Forward Neural Network," JCIT, vol. 1, pp. 64 - 68, 2010.

N. Qian and T. J. Sejnowski, "Predicting the Secondary Structure of Globular Proteins Using Neural Network Models," J. Mol. Biol. 202, pp. 865 - 884, 1988.

M. F. Moller, "A Scaled Conjugate Gradient Algorithm for Fast Supervised Learning," Neural Networks, vol. 6, pp. $525-533,1993$.

Jpred 3, http://www.compbio.dundee.ac.uk/www-jpred/

David T.Jones, "Protein Secondary Structure Prediction Based on Position-specific Scoring Matrices," J.Mol.Biol.,vol.292, pp.195-202, 1999.
B. Sepideh, S. S. Ali, and G. A. R, "Pruning neural networks for protein secondary structure prediction," 8th IEEE International Conference on BioInformatics and BioEngineering, 2008. BIBE 2008, pp. 1 - 6, 2008.

K. Rajasekhar, D. V. Kumar, and O. O. Ahmad, " A twostage neural network based technique for protein secondary structure prediction " The 2nd International Conference on Bioinformatics and Biomedical Engineering, 2008. ICBBE 2008, pp. 1355 - 1358 2008. 